\title{
Evidenced-based local anaesthesia-is there a difference in practice between specialties in a major trauma centre?
}

\author{
Edward Paul Joseph Muscat ${ }^{1,2}$ (D) $\cdot$ Stephen Ali $^{2} \cdot$ Juan Enrique Berner ${ }^{3}$
}

Received: 25 July 2020 / Accepted: 9 September 2020 / Published online: 9 January 2021

(C) Springer-Verlag GmbH Germany, part of Springer Nature 2021

\begin{abstract}
Background Local anaesthesia (LA) is used widely in several clinical scenarios in hospital practice. A number of minor trauma wounds are treated under LA at the North Bristol Trust, and on most occasions, these patients are admitted to the emergency department (ED) and treated there. On other occasions, more complex wounds may be treated by the plastic surgery team. The aim of this study is to compare the preferences of LA agents between these departments in cases of minor trauma. The authors also present a narrative review of the literature and determine best practice.

Methods A single-centre clinical practice survey was developed and distributed to both departments which involved two case scenarios, one involving a head injury to the occiput (scenario 1) and one nailed repair (scenario 2) both requiring treatment under LA.

Results In both scenarios ED favoured lidocaine (89\%) whereas the plastic surgery team preferred to mix solutions. In scenario 1 , $89 \%$ of ED participants chose lidocaine $1 \%$, but only $38 \%$ of the plastic surgery team chose it. Forty-two percent of ED chose to use adrenaline but $100 \%$ plastic surgery team chose it. Both teams refrained from using adrenaline in digits, but $23 \%$ of the plastic surgery team chose it. Eighty-four percent of ED chose $25 \mathrm{G}$ needles, and the plastic surgery team's choices varied.

Conclusions This study has shown marked differences in the use of LA between two specialties in a major trauma centre. There is good quality evidence to support the safety of adrenaline use in digits with the addition of sodium bicarbonate to decrease the pain inflicted to the patient upon infiltration of LA. This study serves to raise awareness of the surgical dogma around adrenaline use and provide clinicians with an evidence-based guide to managing minor trauma with LA.
\end{abstract}

Level of evidence: Not ratable.

Keywords Local anaesthetic $\cdot$ Adrenaline $\cdot$ Plastic surgery

Edward Paul Joseph Muscat

edward.j.muscat@gmail.com

Stephen Ali

stephenrahemali@gmail.com

Juan Enrique Berner

jeberner@gmail.com

1 North Bristol Trust, Southmead Road, Bristol BS10 5NB, UK

2 Morriston Hospital, Heol Maes Eglwys, Swansea SA6 6NL, UK

3 Newcastle Upon Tyne Hospitals NHS Foundation Trust, Newcastle, UK

\section{Introduction}

The use of local anaesthesia (LA) is applied to various clinical scenarios in hospital practice. The choice of agent and its mode of administration are influenced by the clinical scenario faced, clinician experience and knowledge of the best available evidence. The use of LA is invaluable for minor trauma as it facilitates rapid treatment of such wounds and can expedite early discharge. In this era of COVID-19, there is an additional emphasis on clinicians to reduce hospital admission rates and manage patients on a see and treat basis where possible. Furthermore, the $20.5 \%$ mortality rate associated with undergoing surgery with general anaesthesia (GA) during the incubation period of COVID-19 infection means that avoiding GA has never been more vital [1]. The vast majority of patients presenting to the emergency department (ED) can have their minor trauma managed on a see and treat basis by the 
assessing emergency medicine physicians. On-site plastic surgery support may be available for more complex wounds, but for the vast majority of ED cases requiring plastic surgery input, referral to a supraregional centre will be required. In both settings, the choice of LA and method of delivery is important for adequate procedural anaesthesia in order to prevent procedural failure which may lead to an avoidable referral to a distant supraregional centre. Use of short acting agents alone may not provide the adequate duration of anaesthesia for certain procedures. Additionally, consideration of using adrenaline as an adjunct, a longer-acting LA or even a combined mixture of LA could provide a more effective analgesic effect and an increased duration of LA depending on the procedure. LA is acidic in nature, and if delivered inappropriately at high pressure and over a short time, it can be a painful for the patient. This is unacceptable and good technique will improve the experience for the patient. There is evidence to suggest that the use of sodium bicarbonate acts as a buffer for LA and, by using the appropriate needle gauge, may reduce pain levels. The primary aim of this study is to compare the preferences of local anaesthetic agents between plastic surgeons and emergency physicians in cases of minor trauma. The secondary aim is to present a narrative review of the literature and determine best practice.

\section{Methods}

We conducted a single-centre clinical practice survey over a 3month period. The goal was to compare the different choices of LA agents and techniques between plastic surgeons and emergency physicians at Southmead Hospital, North Bristol NHS Trust a major trauma centre (MTC) in the southwest of England. A questionnaire was designed and distributed amongst staff ranging from senior doctors and specialist nurses of both teams from their respective departments. The questionnaire included two clinical scenarios. Case 1 involved a scenario where a patient suffered a fall to his occiput and sustained a horizontal $4 \mathrm{~cm}$ by $0.5 \mathrm{~cm}$ clean wound that required suturing and haemostasis. Case 2 described a patient who suffered a nail bed injury of the right middle finger who required repair under LA.

The questions for both cases requested information about LA choice, adjuncts, size of needle, volume of LA, use of adrenaline and site of infiltration in order to treat the injuries. The responses were reviewed and compared with evidencebased practice.

The chi-square $\left(X^{2}\right)$ test was used post hoc to test the hypothesis of no difference in intra- and intergroup use of type of LA, sodium bicarbonate, adrenaline, size of syringe, volume of LA used and needle calibre. Statistical analysis was performed using GraphPad Prism 8.0 (La Jolla, CA, USA). $P<0.05$ was taken as significant.

\section{Results}

A total of 36 participants were included. Twenty (55\%) participants from the ED team which included nurse practitioners $(n=5)$, senior house officers (postgraduate years 2-4) $(n=7)$, specialist registrars (postgraduate years 5-8) $(n=6)$ and consultants $(n=2)$. There were sixteen $(45 \%)$ participants from the plastic surgery team which included core trainees (postgraduate years $3-4)(n=8)$, specialist registrars (postgraduate years 5-8) $(n=7)$ and consultants $(n=1)$.

\section{Choice}

In both scenarios, respondents from the ED favoured lidocaine $1 \%$ as their preferred choice whereas plastic surgery respondents preferred to mix solutions of short acting (lidocaine $1 \%$ or $2 \%$ ) and long acting LA (bupivacaine $0.5 \%$ ) together (50:50). In case $1,89 \%$ of the ED chose lidocaine $1 \%$. Interestingly, only $38 \%$ of the plastic surgery team chose to use lidocaine $1 \%$ alone. In case 2, 66\% of the ED opted for lidocaine where $46 \%$ of plastic surgeons chose to mix their LA solutions (lidocaine and bupivacaine).

\section{Adrenaline}

In case 1 , only $42 \%$ of ED respondents chose to use adrenaline whereas $100 \%$ of the plastic surgery respondents chose it to manage the scalp wound. Both teams refrained from using adrenaline in case 2 as it involved a digit $(23 \%$ of plastic surgery respondents and $5 \%$ of ED respondents opted for the inclusion of adrenaline).

\section{Sodium bicarbonate}

Both teams generally refrained from using sodium bicarbonate $(0 \% \mathrm{ED}$ and $8 \%$ plastic surgeons in case $1 ; 5 \% \mathrm{ED}$ and $8 \%$ plastic surgeons in case 2).

\section{Volume of LA}

Respondents from ED opted to draw up less volume as opposed to plastic surgeons. In case 1, 52\% of ED chose to draw up $5 \mathrm{~mL}$ whereas $77 \%$ plastic surgeons chose $10 \mathrm{~mL}$ volumes of LA. In case 2, the majority of ED, $52 \%$, opted for $2.5 \mathrm{~mL}$ whereas $85 \%$ plastic surgeons chose $10 \mathrm{~mL}$.

\section{Needles}

ED staff generally chose smaller gauged needles; however, the plastic surgery respondents' choices were more varied. In case 1 , a large majority of $\mathrm{ED}, 84 \%$, chose orange needles $(25 \mathrm{G})$ for the scalp wound where plastic surgeons chose several types (38\% grey ( $27 \mathrm{G}), 31 \%$ blue $(23 \mathrm{G}), 23 \%$ 
orange $(25 \mathrm{G})$ ). In case $2,68 \%$ of ED chose orange needles when approaching the injured digit whereas plastic surgeons chose a variation of needles ( $42 \%$ grey, $25 \%$ blue, $33 \%$ orange).

\section{Site}

Both teams proposed a scalp block and a ring block in cases 1 and 2 respectively.

\section{Post hoc statistical analysis}

Despite these observed clinical differences in LA type, sodium bicarbonate, adrenaline, size of syringe, volume and needle calibre used between the ED and plastic surgery teamsthere was no statistically significant difference relating to these practices stratified by intra- and intergroup training levels.

\section{Discussion}

Local anaesthetic agents are chemicals that reversibly block intracellular voltage-gated sodium channels. They cause the abolition of conduction of nerve impulses by increasing the threshold for excitation in the nerve, by slowing the propagation of the nerve impulse, thereby, reducing the development of the action potential [2]. With hand injuries and facial lacerations alone making up nearly a third of all ED attendances, the use of LA in the management of trauma is commonplace for any ED or plastic surgery trauma service. It is therefore of paramount importance that evidence-based practice underpins LA use to improve analgesic efficacy, reduce procedural failure and conversion to GA and finally reduce pain during the initial administration.

The results of this study show differences in practice amongst the emergency and plastic surgery departments, in a MTC, in their use of local anaesthetic in the management of hand and craniofacial trauma. This signifies that despite availability of published evidence on indications for local anaesthetic use and strategies to mitigate pain, there are still differences regarding its application. This has consequences on how easily the procedure is carried out and on patients' experiences.

The most commonly used LA agents in practice are lidocaine and bupivacaine. The onset of action of lidocaine is rapid at about 45-90 s; however, alone, it is short acting with a duration of 10-20 min. Therefore, lidocaine is suitable for infiltration, block and surface anaesthesia. On the other hand, bupivacaine has a much slower onset of action at about 510 min after injection but has a longer duration of action at 4 $8 \mathrm{~h}$ [3]. A dilute concentration of adrenaline $(1: 200,000)$ usually reduces absorption capability and concentration of bupivacaine. This would allow the use of larger maximum doses which can prolong the duration of action. Due to its slow onset, it is not an ideal sole agent for procedural analgesia. However, combining these two amide LA agents in one syringe offers the clinician and patient the best effects of both drugs: the very rapid onset of lidocaine and the prolonged duration of bupivacaine [4]. Regarding the combined use of dual LA agents into the same infiltration, Ribotsky et al. [5] stated that there is no significant advantage, with respect to onset and duration of local blockade, to using a 50/50 mixture of plain lidocaine and bupivacaine in place of their independent use in his double-blinded trial. These findings were also demonstrated by Valvano and Leffler [6] who stated that it is not necessary to use lidocaine/bupivacaine to achieve faster onset of anaesthesia. They stated that a bupivacaine digital block induces anaesthesia in the same period of time and with equivalent pain of injection as a 1:1 lidocaine/bupivacaine combination. This concept is theoretical without much literature supporting this. With regards to bupivacaine alone, Thomson and Lalonde [7] found that bupivacaine produces twice the duration of anaesthesia than lidocaine with adrenaline. Mixing is not recommended by pharmaceutical companies, but its practice is still preferred by some specialities [7]. From our study, the majority of the plastic surgery team chose to mix their LA solutions. The safest practice would be to refrain from achieving the total maximum mixed LA doses in order to avoid LA toxicity. However, little evidence exists as to whether mixing LA solutions augments or decreases its performance [7].

\section{Adrenaline use in the scalp}

The vasoconstriction effect of adrenaline in managing scalp wounds has the benefit of reducing bleeding which improves visualisation of the surgical field and it slows the local washout of LA which has the benefit of prolonging its action. It was interesting to note the differences with regards to adrenaline use amongst both teams (100\% plastic surgery respondents and 43\% ED respondents). Injuries to the scalp can be anaesthetised by performing a posterior scalp block. The greater occipital nerve can be blocked by injecting LA midway between the occipital protuberance and the mastoid process, $2.5 \mathrm{~cm}$ lateral to the nuchal median line. Either that or a wound in the area of the scalp can be infiltrated with LA around its margins. The scalp is well vascularised, and wounds over that area surmount to significant bleeding. Accidental LA injection has been associated with acute rises in LA plasma concentration, which may result in LA toxicity. Evermore so, the use of adrenaline may thus especially be recommended in well vascularized areas such as the scalp to maximise block duration, minimise bleeding and decrease the risk 
of having acute rises in the plasma concentration of LA [8].

\section{Adrenaline use in the digits}

In our study, few plastic surgeons chose to use adrenaline in combination with LA for a digital block, and almost none of the emergency physicians opted to use it. However, local anaesthetic in digits can be helpful for cases as it can avoid tourniquet use and associated tourniquet pain if used more proximally. Level II evidence has demonstrated that tourniquet pain hurts more than LA injection pain with adrenaline hemostasis justifying its use in this context [9, 10]. "Adrenaline should never be injected into the finger, because of the gangrene that frequently results." Such a statement appears in Bunnel's textbook Surgery of the Hand (1956) which is where the practice of adrenaline in association with a local anaesthetic has been avoided and persists to this day for fear of digit necrosis [11]. This dogma has spread and is rooted in the teachings in medical schools, where it was common to teach that adrenaline should not be injected into the extremities (fingers, nose, penis, and feet) [11]. Evidence-based medicine has subsequently overturned this surgical dogma. Despite this, both specialities preferred to refrain from supplemental adrenaline use in the digits. The culture of not injecting adrenaline into fingers commenced sometime between 1920 and 1940, during the time when procaine was used which resulted finger necrosis with or without adrenaline. Nearly all of the 48 reported cases of finger necrosis attributable to procaine local anaesthesia occurred before 1950, with most implicating cases involved procaine without adrenaline. Procaine is a LA which is quite acidic, with a $\mathrm{pH}$ of 3.6 , which can acidify to a $\mathrm{pH}$ of 1 with prolonged storage. It is likely that the acidic nature of this LA would be the causative agent for the historical reports of finger necrosis and not due to the combined effect of adrenaline with it $[12,13]$. Twenty-one cases of digital necrosis with adrenaline as well as a LA have been reported. Most of which occurred before 1950, and all involved the use of procaine or cocaine which could have been expired, therefore, had an extremely low $\mathrm{pH}$ [12]. Thomson et al. [14] reported that this could have led to cause of the digital necrosis as it becomes more acidic with age. Phentolamine is a reversible nonselective alpha 1 blocker [15] which can reverse the effects of adrenaline in the digits. None of these cases had a phentolamine rescue.

There is sufficient evidence to indicate that digital blocks with adrenaline are not only safe but a very useful adjunct in hand surgery. Fitzcharles-Bowe et al. adds to the argument with his review on 59 patients who suffered accidental injections of highly concentrated adrenaline $(1: 1,000)$ into the fingers, none who suffered digital necrosis [16]. Lidocaine, by contrast, has been used safely both with and without adrenaline. An extensive review of the literature from 1880 to 2000 revealed no documented cases of finger necrosis resulting from LA with lidocaine plus adrenaline [17]. In their series of 3100 patients, Lalonde et al. observed no ischemia or necrosis in the wounds or in the fingers when adrenaline 1:100,000 or less was used in combination with LA in the elective setting [18]. In the uneventful case of vascular compromise, it is important to remember that phentolamine exists as a rescue agent. In a systematic review on patients with cardiac comorbidities, it is found that in $2 \%$ lidocaine with $1: 100,000$ adrenaline, only $20 \%$ of patients experienced tachycardias and $15 \%$ had arrhythmias [19]. Therefore, despite its safety in respect to tissue viability, it is important to warn the patient about the risk of palpitations or an 'adrenaline rush'.

\section{Use of a buffering agent}

Alkalinisation of LA has its potential advantages. The higher $\mathrm{pH}$ of the solution may result in reduced 'burning' pain experienced by the patient. Also, after infiltration, the $\mathrm{pH}$ of this solution may achieve normal tissue $\mathrm{pH}$ more rapidly. A recent metaanalysis and systematic review concluded that sodium bicarbonate buffered with 1:10 lidocaine was associated with a statistically significant pain reduction compared with unbuffered lidocaine. A Cochrane review [20] quoted that the correct concentration of bicarbonate necessary to effectively buffer the solution and raise the solution $\mathrm{pH}$ to a physiologic 7.4 is a $1: 10$ ratio of $8.4 \%$ sodium bicarbonate to $1 \%$ lidocaine with $1: 100,000$ adrenaline. However, other studies did show that adrenaline is chemically unstable in LA solutions alkalinised with sodium bicarbonate used as a buffering agent [21]. In an article reviewing epidural anaesthesia preparations, those mixtures alkalinised with sodium bicarbonate showed that the adrenaline concentrations effectively decreased over $24 \mathrm{~h}$, whereas those in nonalkalinised mixtures did not change with time. Bupivacaine and lidocaine concentrations in all mixtures did not decrease over time regardless of alkalinisation. Frank et al. reported that $1 \%$ of low-dose adrenaline was found to be one thousand times more acidic than subcutaneous tissue. He demonstrated that adrenaline solutions had a $\mathrm{pH}$ of 4 whereas lidocaine without adrenaline had a $\mathrm{pH}$ of 6 [22]. The outcome of a study by Phero et al. indicated markedly reduced pain levels inflicted on injections into the skin with buffered lidocaine. Seventy-eight percent of the 28 subjects reported either a reduction or the similar level of pain scores with the buffered drug. Sixty-one percent of subjects reported a shorter time to lower lip numbness with the buffered drug [23]. 


\section{Needle gauge}

Gauge refers to the diameter of the lumen of the needle, therefore the smaller the number, the greater the diameter of the lumen. A 30-gauge needle has a smaller internal diameter than a 25-gauge needle, for example. Hamburg [24] was able to demonstrate that patients are unable to ascertain differences among 23-, 25-, 27-, and 30-gauge needles. Gill et al. was able to show that the insertion of a 27 - or 28-gauge needle had a $50 \%$ likelihood of being reported as painful, which was marginally greater than that of a 31-gauge needle, causing $39 \%$ likelihood of pain received [25]. When choosing a needle gauge, the deflection along the bevel must also be taken into consideration. Therefore, the smaller the diameter of the needle, the higher the chance of it causing deflection off its planned path. Reed et al. [26] indicated that 30-gauge needles result in considerable deflection along its axis, whereas 25gauge needles essentially do not deflect at all. It is well documented that the larger diameter needle, the more pain is inflicted upon injection which is why needle gauge should be carefully considered before administering LA. Kobayashi et al. found that the administration of $2-\mathrm{ml} 1 \%$ lidocaine with a 24-gauge needle was more painful than $0.5 \mathrm{ml} 1 \%$ lidocaine with a 27-gauge needle [27]. The direction of needle tilt during infiltration is often overlooked, but there is level II evidence showing that putting the needle in at $90^{\circ}$ hits fewer nerves and hurts less than at $45^{\circ}$. As soon the needle has entered the skin infiltrating $5 \mathrm{cc}$ under the dermis and not within it, level II evidence has been shown it reduces pain $[28,29]$.

\section{Limitations}

- This was a single-centre and not a multicentre study.

- The patient's pain perspective was not reviewed.

\section{Conclusions}

This study has shown marked differences in the use of LA practice between two specialties in a MTC. To minimise pain experienced by the patient, we should do our utmost to develop a better understanding of the properties of what medication we are infiltrating and the techniques on how to mitigate this. There is good quality evidence to support the safety of adrenaline use in digits whilst the addition of sodium bicarbonate and use of smaller needle is commensurate with its proven ability to decrease the pain inflicted to the patient upon infiltration of LA. We hope this study serves to raise awareness of the surgical dogma around adrenaline use and that it provides clinicians with an evidence-guide to managing minor trauma with LA whilst improving the experience for patients.

Acknowledgments The authors would like to thank both the Emergency Department and the Plastic Surgery of the North Bristol Trust for their help in this study.

\section{Compliance with ethical standards}

Conflict of interest Edward Muscat, Stephen Ali and Juan Enrique Berner declare no conflict of interest.

Ethical approval This is survey-based study. The local Research Ethics Committee has confirmed that no ethical approval is required.

Informed consent Informed consent was obtained from all individual participants included in the study.

\section{References}

1. Lei S, Jiang F, Su W et al (2020) Clinical characteristics and outcomes of patients undergoing surgeries during the incubation period of COVID-19 infection. EClinicalMedicine 21:4f

2. Balakrishnan K, Ebenezer V, Dakir A et al (2015) Bupivacaine versus lignocaine as the choice of locall anesthetic agent for impacted third molar surgery a review. J Pharm Bioallied Sci 7(Suppl 1): S230-S233

3. https://clinicaltrials.gov/ct2/show/NCT01751347. Date accessed: $30 / 03 / 2020$

4. French J, Sharp LM (2012) Local anaesthetics. Ann R Coll Surg Engl 94(2):76-80

5. Ribotsky BM, Berkowitz KD, Montague JR (1996) Local anesthetics. Is there an advantage to mixing solutions? J Am Podiatr Med Assoc 86(10):487-491

6. Valvano MN, Leffler S (1996) Comparison of bupivacaine and lidocaine/bupivacaine for local anesthesia/digital nerve block. Ann Emerg Med 27(4):490-492

7. Thomson CJ, Lalonde DH (2006) Randomized double-blind comparison of duration of anesthesia among three commonly used agents in digital nerve block. Plast Reconstr Surg 118(2):429-432

8. Osborn I, Sebeo J (2010) Scalp Block" during craniotomy: a classic technique revisited. J Neurosurg Anesthesiol 22:187-194

9. Braithwaite BD, Robinson GJ, Burge PD (1993) Haemostasis during carpal tunnel release under local anaesthesia: a controlled comparison of a tourniquet and adrenaline infiltration. J Hand Surg (Br) 18(2):184-186

10. Ralte P (2010) Haemostasis in open carpal tunnel release: tourniquet vs local anaesthetic and adrenaline. Open Orthop J 4:234

11. Novais Junior RA, Costa JRB, Carmo JM (2014) Use of adrenalin with lidocaine in hand surgery. Rev Bras Ortop 49(5):452-460

12. Neto PJ, Moreira L, Casas PP (2017) Is it safe to use local anesthesia with adrenaline in hand surgery? WALANT technique. Rev Bras Ortop 52(4):383-389

13. Lalonde D, Martin A (2013) Epinephrine in local anesthesia in finger and hand surgery: the case for wide-awake anesthesia. J Am Acad Orthop Surg 21:443-447

14. Thomson CJ, Lalonde DH, Denkler KA et al (2007) A critical look at the evidence for and against elective epinephrine use in the finger. Plast Reconstr Surg 119:260-266

15. Brock G (2000) Oral phentolamine (Vasomax). Drugs Today (Barcelona) 36(2-3):121-124 
16. Fitzcharles-Bowe C, Denkler K, Lalonde D (2007) Finger injection with high-dose $(1: 1,000)$ epinephrine: does it cause finger necrosis and should it be treated? Hand. 2(1):5-11

17. Mohan PP, Cherian PT (2007) Epinephrine in digital nerve block. Emerg Med J 24(11):789-790

18. Lalonde D, Bell M, Benoit $\mathrm{P}$ et al (2005) A multicenter prospective study of 3,110 consecutive cases of elective epinephrine use in the fingers and hand: the Dalhousie Project Clinical Phase. J Hand Surg [Am] 30(5):1061-1067

19. Godzieba A, Smektala T, Jędrzejewski M et al (2014) Clinical assessment of the safe use local anaesthesia with vasoconstrictor agents in cardiovascular compromised patients: a systematic review. Med Sci Monit 20:393-398

20. Cepeda MS, Tzortzopoulou A, Thackrey M, Hudcova J, Arora Gandhi P, Schumann R (2010) Adjusting the $\mathrm{pH}$ of lidocaine for reducing pain on injection. Cochrane Database Syst Rev (12): CD006581

21. Robinson J, Fernando R, Sun Wai WY et al (2000) Chemical stability of bupivacaine, lidocaine and epinephrine in $\mathrm{pH}$-adjusted solutions. Anaesthesia 55:853-858

22. Frank SG, Lalonde DH (2012) How acidic is the lidocaine we are injecting, and how much bicarbonate should we add? Can J Plast Surg 20:71-74
23. Phero JA, Nelson B, Davis B et al (2017) Buffered versus nonbuffered lidocaine with epinephrine for mandibular nerve block: clinical outcomes. J Oral Maxillofac Surg 75(4):688-693

24. Hamburg HL (1972) Preliminary study of patient reaction to needle gauge. N Y State Dent J 38(7):425-426

25. Gill HS, Prausnitz MR (2007) Does needle size matter? J Diabetes Sci Technol 1(5):725-729

26. Reed KL, Malamed SF, Fonner AM (2012) Local anesthesia part 2: technical considerations. Anesth Prog 59(3):127-137

27. Kobayashi Y, Kamada Y, Ichimiya T, Namiki A (1999) Is local anesthesia necessary for spinal needle insertion? Masui:1033-1036

28. Martires KJ, Malbasa CL, Bordeaux JS (2011) A randomized controlled crossover trial: lidocaine injected at a 90-degree angle causes less pain than lidocaine injected at a 45-degree angle. J Am Acad Dermatol 65:1231-1233

29. Arndt KA (1983) Minimizing the pain of local anesthesia. Plast Reconstr Surg 72:676-679

Publisher's note Springer Nature remains neutral with regard to jurisdictional claims in published maps and institutional affiliations. 